\title{
Como o Design Thinking é ensinado nas melhores universidades do mundo?
}

How is Design Thinking taught in world's best universities?

CANFIELD, Daniel de Salles; Universidade Federal do Rio Grande do Sul

daniel@4sc.com.br

ABREU, Tiago Moreira de; Universidade Federal do Rio Grande do Sul

tiago.abreu@ufrgs.br

VAN DER LINDEN, Júlio Carlos de Souza; Universidade Federal do Rio Grande do Sul

julio.linden@ufrgs.br

BERNARDES, Mauricio Moreira e Silva; Universidade Federal do Rio Grande do Sul

bernardes@ufrgs.br

\section{Resumo}

Esta pesquisa tem como propósito analisar como as 20 melhores universidades do mundo estão ensinando o Design Thinking (DT). Por meio de uma pesquisa documental, no website das instituições, foram classificadas as práticas pedagógicas que abordam o tema, compreendidas por localidade, tipo de prática pedagógica, público-alvo, programa de ensino, professores e sua linha de pesquisa. Verificou-se que, de fato, o Design Thinking é ensinado em todas as universidades. Para as 96 práticas pedagógicas encontradas, 49 se encontra o DT no título da prática e 47 nos resumos. Verificou-se uma tendência de que os professores (62\%) tenham contato direto com o mercado, seja como empreendedor ou profissional.

Palavras Chave: Design Thinking; Ensino; Universidades e Pesquisa documental.

\begin{abstract}
This research aimed at analyzing how Design Thinking (DT) has been taught in the top 20 ranked universities in the world. The study was carried out through a documentary research, based on the websites of these specific institutions. The pedagogical approaches to the theme were classified according to the following criteria: the location, the type of pedagogical practice, the target audience, the teaching program, the professors, and the research line. Design Thinking has been found to be taught indeed in all the universities checked. Taking under consideration the 96 courses found, 49 presented DT in their title, and 47 in their syllabus. A tendency for professors (62\%) to have direct market experience, either as an entrepreneur or as a professional, was also observed.
\end{abstract}

Keywords: Design Thinking; Teaching; Universities and Documentary research. 


\section{Introdução}

A evolução do conhecimento sobre as práticas do designer vem rendendo frutos para metodologias. O Design Thinking (DT) se apresenta como uma metodologia capaz de ser aplicada por diversas escolas, idades e formações. Isto dá a este processo cognitivo uma flexibilidade para se adaptar à realidade. Desde seu surgimento até os dias de hoje, o design está em constante mudança e evolução. A metodologia está ganhando território como um elemento estratégico, deixando de atuar simplesmente como uma ferramenta operacional. De acordo com Simon (1996), o design é um conhecimento comum para todas as áreas e seu papel é apoiar qualquer profissional para resolver um problema.

Portanto, o objetivo desta pesquisa documental é identificar como as 20 melhores universidades do mundo empregam o DT no contexto da educação. É relevante realizar esta pesquisa, uma vez que o DT e a educação são tópicos trabalhados no mundo acadêmico, para proporcionar uma visão original e sugerir novas lacunas de estudos e direções. Além disso, o aspecto interdisciplinar que envolve o assunto do DT expande seu uso além dos limites do design, tornandoo um ponto de interesse valioso em muitas outras áreas do conhecimento.

O estudo apresenta o termo DT, definições e principais autores conforme sua evolução histórica em seu referencial teórico. Após, é apresentado o método de pesquisa, base de dados e uma discussão destes dados. Neste caso, uma pesquisa documental é apontada descrevendo o protocolo de estratégia de busca baseado em Dresch, Lacerda e Antunes Jr (2015). Finalmente há a conclusão do estudo por meio de uma análise dos achados nas 20 universidades.

\section{Design Thinking}

O Design Thinking é considerado uma abordagem centrada no ser humano. A maneira de pensar do designer, cria soluções de forma colaborativa que utiliza a sensibilidade e os métodos do designer para converter a necessidade em demanda (TSCHIMMEL, 2012). O estudo dos processos cognitivos do designer remonta sua origem na década de 1950 e é continuamente explorado ao longo das décadas (Osborn em 1953, Bono em 1967, Simon em 1969, Koberg e Bagnall em 1972, Rittel e Webber em 1973 e Cross in 1982).

Blanco (1985) argumenta que uma das características fundamentais da maneira de pensar do designer é a lógica abdutiva (em contrapartida com a lógica indutiva) (apud ORTHEL, 2015). Dorst (2011) acredita que designers vem desenvolvendo também a capacidade de abstração ao longo de sua história. No entanto, Owen (1998) acredita que o pensamento abdutivo não é autossuficiente, mas que durante o desenvolvimento da solução, ou do produto, o profissional deve ter a capacidade de oscilar entre abdução e concreto, e entre análise e síntese.

O termo Design Thinking foi sugerido por Peter Rowe em seu livro intitulado "Design Thinking". O autor defendeu que os designers têm uma maneira única de abordar o seu trabalho, um processo único de solução de problemas (KIMBELL, 2011; LUPTON, 2011; TSCHIMMEL, 2012, 2014; WOUDHUYSEN, 2011). Seguindo o mesmo conceito de DT, um grupo de pesquisadores (Cross, Dorst, Roozenburg, Lawson, Eastman, McCracken e Newstetter) continuaram a estudá-lo focado no processo cognitivo dos designers: "identificar as estratégias mentais essenciais dos designers enquanto trabalhavam em um projeto" (TSCHIMMEL, 2012, p. 2).

Além disso, Richard Buchanan adicionou um segundo conceito holístico do DT com o artigo Wicked Problems in Design Thinking (1992) baseado em abordagem de problemas "mal 
estruturados" formulada por Horst Rittel na década de 60. A descrição do DT de Buchanan está preocupada em "conectar e integrar conhecimentos úteis das artes e ciências [...] de maneiras adequadas aos problemas e propósitos do presente".

Recentemente, Johansson-Sköldberg, Woodilla E Çetinkaya (2013) por meio de uma revisão sobre o termo Design Thinking, sugerem que seja classificado de duas maneiras: o Designerly Thinking, com um viés acadêmico, interessado, primeiramente, na criação de artefatos, depois como uma prática reflexiva; e o Design Thinking com um viés de gestão, focado na maneira de trabalhar em equipe e como abordar problemas.

Roger Martin, professor do Harvard Business Institute, acredita no DT como um processo, que pode ser utilizado para o redesenho contínuo de uma empresa (LEAVY, 2010). Uma vez que há um mistério, ou seja, não é possível compreender o problema na sua totalidade, devem haver heurísticas que se adequem a realidade, sendo estas selecionadas e aprimoradas para a criação de algoritmos. Tudo isso por meio do DT. Considera também uma maneira de aproximar escolas clássicas como a administração às artes (MARTIN, 2009).

Estas práticas do DT, focam na experiência do usuário, entre elas a parte emocional também, incentivam modelos, como diagramas e esboços para descrever problemas complexos; exploraram o potencial das soluções por meio de protótipos, não encorajam a falha, mas a toleram. $E$ este processo se reforça por meio da iteração.

A aplicabilidade do DT pode ser estendida para diversas áreas, no entanto não para todo tipo de problemas. Liedtka e Ogilve (2011), que focam suas aplicações do processo para gestores, sugerem uma série de quatro perguntas (quadro 1) para compreender se o problema passível de ser resolvido por DT ou, caso contrário, por uma metodologia de resolução de problemas mais linear.

Quadro 1 - Perguntas que auxiliam no entendimento sobre se o problema é wicked ou linear

\begin{tabular}{|c|c|c|}
\hline Pergunta & Design Thinking é mais apropriado & $\begin{array}{l}\text { Uma abordagem linear é mais } \\
\text { apropriada }\end{array}$ \\
\hline $\begin{array}{l}\text { Este problema é centrado } \\
\text { no ser humano? }\end{array}$ & $\begin{array}{l}\text { É necessário um grande envolvimento dos } \\
\text { usuários para compreender }\end{array}$ & $\begin{array}{l}\text { Existem poucos seres humanos } \\
\text { envolvidos no problema ou na solução }\end{array}$ \\
\hline $\begin{array}{l}\text { Quão claro o problema é } \\
\text { compreendido? }\end{array}$ & $\begin{array}{l}\text { É necessário explorar e chegar em um } \\
\text { consenso sobre qual o verdadeiro } \\
\text { problema }\end{array}$ & $\begin{array}{l}\text { O problema é claramente } \\
\text { compreendido que existe apenas uma } \\
\text { solução }\end{array}$ \\
\hline Qual o ní & $\begin{array}{l}\text { Existem diversos fatores desconhecidos e } \\
\text { os dados do passado podem não ser úteis }\end{array}$ & $\begin{array}{l}\text { É possível extrapolar dados passados } \\
\text { para prever o futuro. }\end{array}$ \\
\hline Quais o & $\begin{array}{l}\text { Há pouca informação relevante sobre o } \\
\text { assunto }\end{array}$ & $\begin{array}{l}\text { Existem diversas abordagens e dados } \\
\text { análogos que podem ser utilizadas }\end{array}$ \\
\hline
\end{tabular}

Fonte: Liedtka, Ogilvie e Brozenske (2014), adaptado pelos autores

Versátil não somente para negócios, o DT tem se mostrado eficaz para a resolução de problemas em diversas áreas como: Sustentabilidade (ANDREWS, 2015; SHAPIRA; KETCHIE; NEHE, 2017; BIRKELAND, 2012); novos negócios (MUELLER; THORING, 2012; KATHERINE, 2016); Inovação (LIEDTKA, 2011; BONINI; SBRAGIA, 2011); Educação (MELLES; HOWARD; THOMPSON-WHITESIDEC, 2012; BEHM; CULVENOR; DIXON, 2014; CASSIM, 2013; BURDICK; WILLIS, 2011; ORTHEL, 2015); saúde (HEIJDE, 2016); entre outros. 
Por ser centrado no problema do usuário, Burdick (2011) considera o DT como uma abordagem de alfabetização adequada ao século 21. Estudos sobre a aplicação de metodologias baseadas no Design Thinking sugerem que não há diferença significativa entre sua aplicação por designer e não designers (ORTHEL, 2015). Uma vez que foca seu aprendizado baseado na ação e na descoberta da solução iterando com as partes interessadas. Durante a execução o uso de ferramentas desde o início do trabalho interdisciplinar, torna sua aplicação quase imediata. Como o conhecimento adquirido é baseado em episódios, mais do que processual, a solução tem um alto grau de autocrítica. Portanto, considera-se plausível que universidades de ponta ao redor do mundo estejam de fato adotando a metodologia.

\section{Procedimentos Metodológicos}

Este trabalho utilizou uma pesquisa documental para identificar, nos websites das 20 melhores universidades do mundo, como o Design Thinking está sendo abordado na educação. De acordo com Prodavov e Freitas (2013) a pesquisa documental é baseada em materiais que não receberam tratamento analítico ainda e que se distingue da pesquisa bibliográfica por não ser oriunda da contribuição de vários autores. Para estruturar a estratégia de busca, o protocolo de pesquisa desenvolvido por Dresch, Lacerda e Antunes Jr (2015, p. 142) foi utilizado pois é "voltado a revisões sistemáticas com fins acadêmicos, podendo ser adaptado a revisões com outras finalidades". Ele está representado no Quadro 2.

Quadro 2 - Protocolo de pesquisa

\begin{tabular}{ll}
\hline Protocolo & \\
\hline Framework conceitual & $\begin{array}{l}\text { Como o Design Thinking é um tópico relativamente novo, é importante identificar como as } \\
\text { principais universidades do mundo estão abordando esta metodologia em seus currículos. }\end{array}$ \\
\hline Contexto & $\begin{array}{l}\text { Websites das } 20 \text { melhores universidades do mundo, de acordo com o QS World University } \\
\text { Ranking. }\end{array}$ \\
\hline Horizonte & Pesquisa realizada até junho de 2017. \\
\hline Correntes teóricas & Duas teorias educacionais do DT: (1) acadêmica e (2) empresarial. \\
\hline Idiomas & Inglês. \\
\hline Questão de revisão & Como as melhores universidades do mundo estão ensinando o Design Thinking? \\
\hline Estratégia de revisão & Revisão agregativa (método dedutivo). \\
\hline Critérios de busca & $\begin{array}{l}\text { Critério de inclusão: (1) possuir a expressão exata no título e/ou no resumo da prática } \\
\text { pedagógica; (2) ainda estar sendo ofertado pela universidade no momento da pesquisa } \\
\text { Critérios de exclusão: (1) não ter acesso as principais informações da prática pedagógica. }\end{array}$ \\
\hline Termos de busca & Expressão exata “Design Thinking". \\
\hline Base de dados & Nas 20 principais universidades do mundo, de acordo com o QS World University Ranking. \\
\hline
\end{tabular}

Fonte: Adaptado de Dresch, Lacerda e Antunes Jr (2015)

\subsection{Framework Conceitual}

A área de interesse desta pesquisa, o DT, ainda é relativamente recente. Diante disso, um panorama atualizado, de como as principais universidades do mundo têm abordado este assunto, torna-se necessário. 


\subsection{Contexto}

Com o intuito de descobrir as principais abordagens educacionais do DT, esta revisão focou nas 20 principais universidades do mundo, de acordo com o QS World University Ranking. Por esta razão, o contexto de busca foi limitado aos websites das universidades: web.mit.edu, www.stanford.edu, www.harvard.edu, www.caltech.edu, www.cam.ac.uk, www.ox.ac.uk, www.ucl.ac.uk, www.imperial.ac.uk, www.uchicago.edu, www.ethz.ch, www.ntu.edu.sg, epfl.ch, www.princeton.edu, www.cornell.edu, www.nus.edu.sg, www.yale.edu, www.jhu.edu, www.columbia.edu, www.upenn.edu e www.anu.edu.au.

\subsection{Horizonte}

Como a intenção da pesquisa foi encontrar práticas pedagógicas (intensivos, workshops, disciplinas, etc.), em andamento, que tem o Design Thinking como tema principal, a pesquisa foi realizada de janeiro de 2017 a junho de 2017 em cada website das principais universidades do mundo.

\subsection{Correntes Teóricas}

Desde o surgimento do Design Thinking, a forma de disseminação desta abordagem e suas ferramentas têm tido duas correntes, uma voltada para a academia (alunos e professores) e outra voltada para o ambiente empresarial. Este estudo levou em consideração estas duas vertentes e buscou identificar como as instituições de ensino superior tem ofertado o DT para estes diferentes públicos.

\subsection{Idiomas}

O idioma utilizado na busca foi o inglês, visto que a identificação das 20 melhores universidades do mundo utilizou como parâmetro a QS World University Ranking, uma instituição global. Igualmente, o idioma norte americano esteve presente em todos os 20 websites das universidades pesquisadas, seja na opção de idioma nativo ou como opção secundária.

\subsection{Questão de Revisão}

Com a intenção de prover um panorama sobre o ensino do DT no mundo, esta pesquisa buscou responder a seguinte questão: Como as melhores universidades do mundo estão ensinando o Design Thinking?

\subsection{Estratégia de Revisão}

A estratégia de revisão agregativa foi escolhida uma vez que as perguntas fechadas foram usadas para testar a teoria (método dedutivo) e os resultados dos estudos revisados foram agregados para responder à questão de revisão. Outro ponto chave desta estratégia é o alcance de extensão de menor amplitude dos websites pesquisados, levando ao uso de uma variedade de estudos primários homogêneos.

\subsection{Critérios de busca}

Os critérios de inclusão obedeceram aos seguintes requisitos: (1) ter a expressão exata ("expressão entre aspas nos índices de busca definidos") no título e/ou no resumo da prática pedagógica (disciplina, intensivo, workshop, etc.); (2) ainda estar sendo ofertado pela universidade no momento da pesquisa (DRESCH; LACERDA; ANTUNES JR, 2015, p. 139). No outro sentido, os critérios de exclusão foram (1) não ter acesso as principais informações da prática pedagógica, como 
descrição, resumo, público-alvo e carga horária.

\subsection{Termos de busca}

Para encontrar práticas pedagógicas focados no DT, o termo de busca foi limitado exclusivamente a este elemento. Portanto, a expressão exata "Design Thinking" foi buscada no título e/ou resumo da prática pedagógica. Posteriormente, uma análise de cada prática encontrada se fez necessária para verificar se o termo encontrado estava sendo utilizado de forma correta. 0 número de estudos encontrados usando os critérios de busca (inclusão e exclusão) é apresentado no quadro 3.

Quadro 3 - práticas pedagógicas por critério de busca

\begin{tabular}{ccccc}
\hline Termo & Inclusão & & Exclusão \\
\hline & Título & Resumo & Total & Faltando informações \\
\hline “Design Thinking” & 49 & 47 & 96 & 7 \\
\hline
\end{tabular}

Fonte: Elaborado pelos autores

\subsection{Base de dados}

Para a eleição das instituições foi utilizado o ranking das 20 melhores universidades do mundo que são apontadas pelo QS World University. Este ranking está em sua décima quinta edição, com 1000 das melhores universidades do mundo, de 85 países diferentes e é construído a partir de dados de mais de 1,2 milhão de trabalhos acadêmicos, 70.000 acadêmicos e 40.000 respostas dos empregadores. Os indicadores utilizados como critério de mensuração são: reputação acadêmica, índice $\mathrm{H}$ (produtividade e o impacto do trabalho publicado), citações por corpo docente e equipe de doutores (QS, 2018). Reconhecemos que outros rankings podem apresentar classificação diferente, ora descartando algumas das instituições aqui eleitas, ora elegendo outras. No entanto, consideramos o ranking da QS por ser a "fonte mais popular mundialmente de dados comparativos sobre o desempenho de universidades" (EXAME, 2018).

\section{Resultados e Discussão}

Após a realização da estratégia de busca, foi realizada a leitura de todos os websites com o objetivo de responder à questão de pesquisa, portanto, seis aspectos foram previamente definidos e identificados em cada prática pedagógica, conforme abordado a seguir.

\subsection{Universidades}

Todas as 20 melhores universidades do mundo, identificadas no ranking da QS World University Ranking (ver quadro 4), possuem ao menos uma prática pedagógica sobre o Design Thinking, ou seja, o DT é ensinado em $100 \%$ das principais instituições de ensino superior do mundo.

Quadro 4 - Ranking das 20 melhores universidades do mundo

\begin{tabular}{llll}
\hline$\#$ & Universidade & $\#$ & Universidade \\
\hline $\mathbf{0 1}$ & Massachusetts Institute of Technology (MIT) & $\mathbf{1 1}$ & Nanyang Technological University \\
\hline $\mathbf{0 2}$ & Stanford University & $\mathbf{1 2}$ & Ecole Polytechnique Fédérale de Lausanne (EPFL) \\
\hline $\mathbf{0 3}$ & Harvard University & $\mathbf{1 3}$ & Princeton University
\end{tabular}




\begin{tabular}{llll}
\hline $\mathbf{0 4}$ & California Institute of Technology (Caltech) & $\mathbf{1 4}$ & Cornell University \\
\hline $\mathbf{0 5}$ & University of Cambridge & $\mathbf{1 5}$ & National University of Singapore (NUS) \\
\hline $\mathbf{0 6}$ & University of Oxford & $\mathbf{1 6}$ & Yale University \\
\hline $\mathbf{0 7}$ & University College London (UCL) & $\mathbf{1 7}$ & Johns Hopkins University \\
\hline $\mathbf{0 8}$ & Imperial College London & $\mathbf{1 8}$ & Columbia University \\
\hline $\mathbf{0 9}$ & University of Chicago & $\mathbf{1 9}$ & University of Pennsylvania \\
\hline $\mathbf{1 0}$ & ETH Zurich & $\mathbf{2 0}$ & The Australian National University \\
\hline
\end{tabular}

Fonte: Elaborado pelos autores

A distribuição geográfica das universidades é limitada a cinco países (figura 1), tendo os Estados Unidos como grande concentrador, com mais da metade das instituições $(n=11)$. Em seguida, a Inglaterra com quatro universidades, Suíça e Singapura com duas universidades cada e Austrália com uma.

Figura 1 - Localização das 20 maiores universidades do mundo

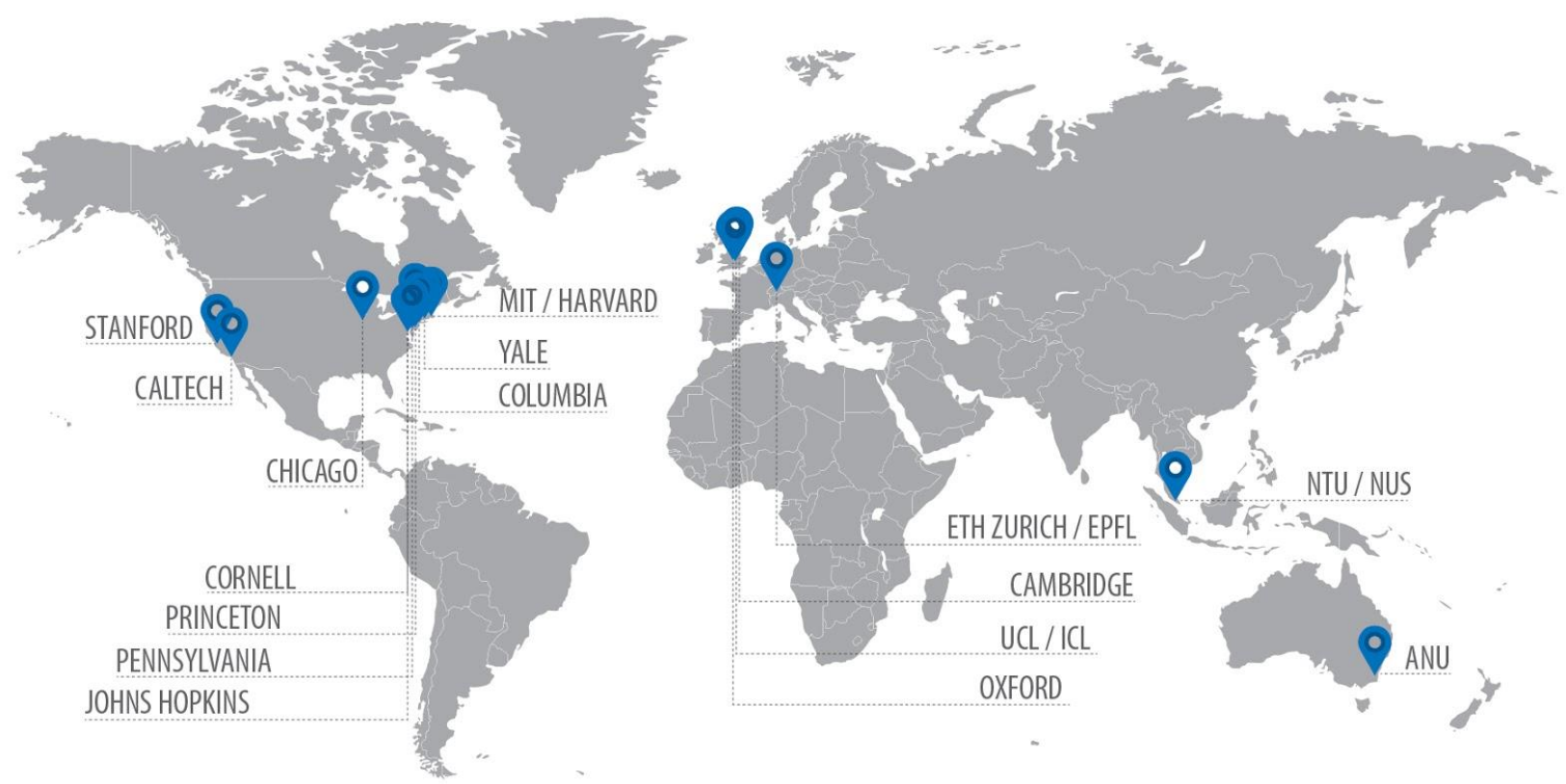

Fonte: Elaborada pelos autores

\subsection{Palavra-chave}

A palavra-chave Design Thinking foi pesquisada nos websites das 20 universidades com a intuito de encontrar práticas pedagógicas que abordem o assunto. $O$ termo DT foi identificado em 96 práticas pedagógicas, sendo 49 nos títulos, como no curso intensivo do MIT intitulado Introduction to Design Thinking for Educators, e em 47 nos resumos, como na disciplina de graduação e pós-graduação Strategy by design da University College London (UCL) que é "[...] introdução ao uso do Design Thinking e da teoria do design como ferramentas na prática da estratégia" (UCL, 2017). 


\subsection{Prática Pedagógica}

A figura 2 mostra a diversidade de formatos que as instituições utilizam para disseminar o Design Thinking. A maior quantidade $(n=41,42,7 \%)$ é através de disciplinas curriculares para alunos de graduação, pós-graduação, professores, entre outros. Em seguida, aparecem formatos com cargas horárias menores, como cursos intensivos $(n=18,18,7 \%)$ de 8 a 36 horas e workshops $(n=14$, $14,5 \%)$ de 2 a 16 horas. $O$ restante é distribuído em bootcamps, programas, laboratório, projetos e outros.

Figura 2 - Tipos de práticas pedagógicas

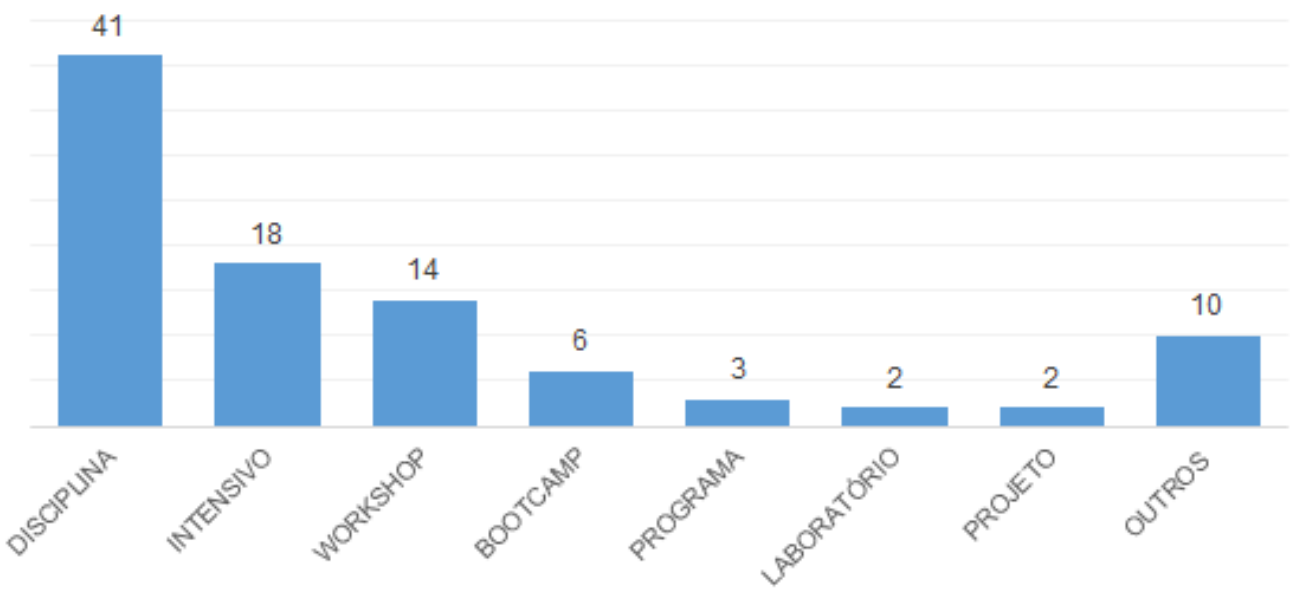

Fonte: Elaborada pelos autores

\subsection{Público-alvo}

O ensinamento do DT nas 20 melhores universidades do mundo é dividido em quatro públicos (figura 3). Como consequência do tipo de prática pedagógica mais adotada, a disciplina, o público-alvo é em sua maioria estudantes $(58,6 \%)$. Sendo estes estudantes pertencentes a diversas categorias, como alunos da 4a série do ensino fundamental, ensino médio, graduação e pósgraduação.

Figura 3 - Práticas pedagógicas por público-alvo

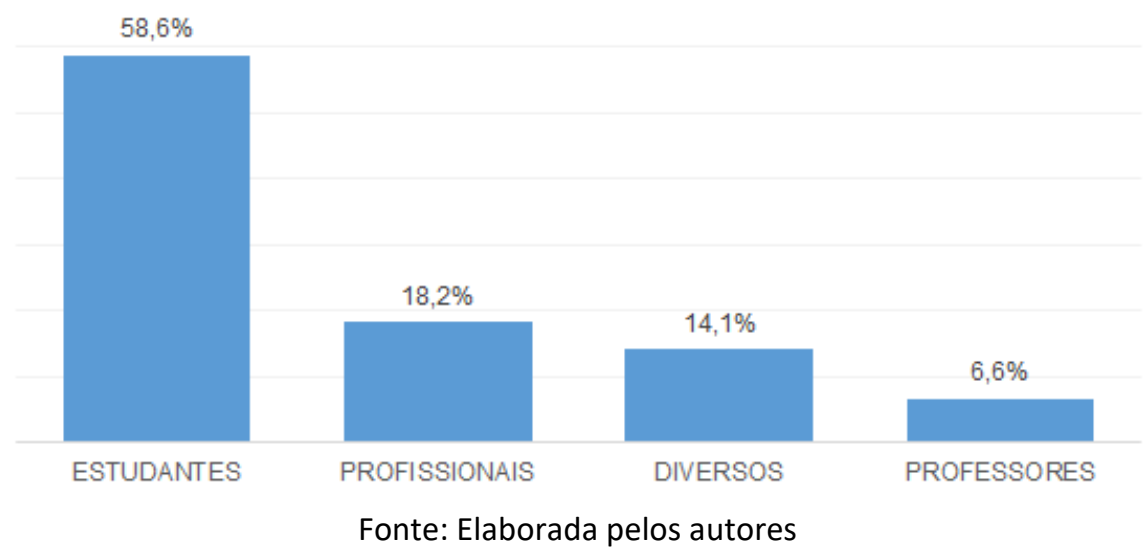


O segundo grande foco é ensinar o DT para o público profissional (18,2\%). As universidades possuem centros de educação executiva que oferecem uma aproximação entre o ambiente acadêmico e empresarial, como no workshop, de dois dias da ETH Zurich, chamado Design Thinking Executive Training. O restante das práticas pedagógicas é voltado para capacitar professores na aplicação do DT e suas ferramentas, e para o público diverso com abordagens mais gerais, como no Design Thinking Bootcamp da University of Cambridge.

\subsection{Programa}

Por ser um assunto relativamente novo e não pertencer exclusivamente a um campo do conhecimento, o ensinamento do DT é ofertado por diferentes programas nas universidades selecionadas (figura 4).

Figura 4 - Práticas pedagógicas por programa

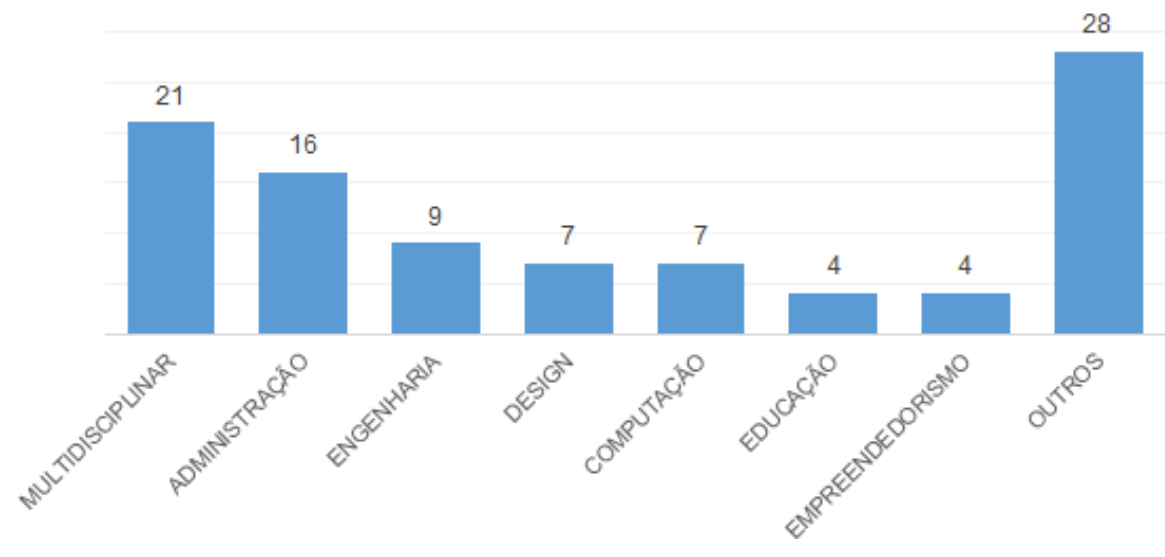

Fonte: Elaborada pelos autores

Consequentemente, o maior número de opções de ensino $(n=21)$ não é pertencente a um programa específico, sendo assim considerado multidisciplinar. Em segundo lugar, a administração é o programa que possui 16 práticas pedagógicas, sendo 10 disciplinas, quatro intensivos, um bootcamp e um clube pertencente a Yale School of management chamado Design + Innovation Club que tem como tema principal o DT.

Diante de sua abordagem ampla, as demais práticas são oferecidas por diversos programas, entre os principais o da engenharia, design, computação, educação e empreendedorismo.

\subsection{Professores}

Um fator que deve ser levado em consideração é o perfil dos profissionais que lecionam o DT nas instituições de ensino (os principais professores pesquisadores e suas linhas de pesquisa são ilustrados no quadro 5).

Observou-se que 62\% dos professores são profissionais do mercado de trabalho, ou seja, possuem ou trabalham em alguma empresa, tendo alguns expoentes do DT como, Lucy Kimbel (sócia da Normann Partners) e David Keley (co-fundador da IDEO). Os outros 38\% dos professores são profissionais exclusivamente dedicados a academia, como Larry Leifer, professor na Stanford University que tem como linha de pesquisa o design engineering. 
Quadro 5 - Principais professores pesquisadores

\begin{tabular}{|c|c|c|c|}
\hline$\#$ & Universidade & Pesquisador & Linha de pesquisa \\
\hline 01 & MIT & Maria Yang & Product Design \\
\hline 02 & Stanford University & $\begin{array}{l}\text { David Keley } \\
\text { Larry Leifer }\end{array}$ & $\begin{array}{l}\text { Creative Confidence } \\
\text { Design Engineering }\end{array}$ \\
\hline 03 & Harvard University & Srikant Datar & Cost Management and Management Control \\
\hline 05 & University of Cambridge & $\begin{array}{l}\text { Marisha Mcauliffe } \\
\text { Victor Seidel }\end{array}$ & $\begin{array}{l}\text { Design Process and DT } \\
\text { Design Process }\end{array}$ \\
\hline 06 & University of Oxford & Lucy Kimbell & Design Thinking and Service Design \\
\hline 08 & Imperial College London & Peter Childs & $\begin{array}{l}\text { Creativity, Product Design, Sustainable Energy and } \\
\text { System Design }\end{array}$ \\
\hline 10 & ETH Zurich & Alan Cabello & $\begin{array}{l}\text { Human-Centered Product, Service and Business } \\
\text { Model Innovation }\end{array}$ \\
\hline 11 & Nanyang Technological University & Wong Yew Leong & $\begin{array}{l}\text { Critical Thinking, Design Thinking and Philosophy of } \\
\text { Education }\end{array}$ \\
\hline 12 & EPFL & Tucci Christopher & $\begin{array}{l}\text { Business Models, Open Innovation and Dynamic } \\
\text { Capabilities }\end{array}$ \\
\hline 14 & Cornell University & Toddi Gutner & $\begin{array}{l}\text { Customer Experience Strategy and Customer- } \\
\text { Focused Innovation }\end{array}$ \\
\hline 15 & National University of Singapore & Jan Auernhammer & Creative Design and Innovation Eco-Systems \\
\hline 19 & University of Pennsylvania & $\begin{array}{l}\text { John Pourdehnad } \\
\text { Natalie W. Nixon }\end{array}$ & $\begin{array}{l}\text { Systems Thinking } \\
\text { Service Design }\end{array}$ \\
\hline
\end{tabular}

Fonte: Elaborado pelos autores

\section{CONSIDERAÇÕES FINAIS}

Este estudo, por meio de uma pesquisa documental, teve o objetivo de responder como as melhores universidades do mundo estão ensinando o Design Thinking. Em primeiro lugar, o DT é ensinado em todas as 20 universidades, ressaltando a atualidade e importância que os principais currículos de ensino superior do mundo prestam a esta abordagem estratégica. Sob o mesmo ponto de vista, ressalta-se que este ensinamento tem dois focos principais, lecionar o DT para estudantes de ensino fundamental a pós-graduação $(58,6 \%)$ através de disciplinas curriculares ( $n=41,42,7 \%)$, e lecionar o DT para profissionais de diversos campos de atuação $(18,2 \%)$ através de práticas pedagógicas com cargas horárias mais curtas, como cursos intensivos ( $n=18,18,7 \%$ ) e workshops ( $n=14,14,5 \%)$. Consequentemente, o perfil dos professores, que lecionam o DT em diversos programas (multidisciplinar, administração, engenharia e outros), é um reflexo do público alvo, ou seja, profissionais tanto do mercado de trabalho (62\% possuem ou trabalham em uma empresa), quanto dedicados somente a carreira acadêmica.

A fim de alcançar um padrão de pesquisa de qualidade, essa investigação teve que considerar suas limitações. Os bancos de dados on-line utilizados não disponibilizam todas as informações necessárias, resultando na exclusão não voluntária de algumas práticas pedagógicas. Acrescenta-se que, os resultados desta pesquisa estão restritos a 20 universidades, de cinco países, desta forma, uma pesquisa futura pode ampliar o número de universidades, acrescentando uma visão mais global sobre o ensinamento do DT. 


\section{Referências}

ANDREWS, D. The circular economy, design thinking and education for sustainability. Local Economy: The Journal of the Local Economy Policy Unit, v. 30, n. 3, p. 305-315, 2015.

BLANCO, H. Pragmatism, abduction, and wicked problems. Berkeley Planning Journal, v. 1, n. 2, p. 93-119, 1985.

BEHM, M.; CULVENOR, J.; DIXON, G. Development of safe design thinking among engineering students. Safety Science, v. 63, p. 1-7, 2014.

BIRKELAND, J. Design blindness in sustainable development: from closed to open systems design thinking. Journal of Urban Design, v. 17, n. 2, p. 167-187, 2012.

BONINI, L. A.; SBRAGIA, R. O modelo de design thinking como indutor da inovação nas empresas: um estudo empírico. Revista de Gestão e Projetos, v. 2, n. 1, p. 03-25, 2011.

BONO, E. De. Why so stupid? Management-Issues, [S.I.], 2006.

BUCHANAN, R. Wicked Problems in Design Thinking. Design Issues, v. 8, n. 2, p. 5-21, 1992.

BURDICK, A.; WILLIS, H. Digital learning, digital scholarship and design thinking. Design Studies, v. 32, n. 6, p. 546-556, 2011.

CASSIM, F. Hands On, Hearts On, Minds On: Design Thinking within an Education Context. International Journal of Art \& Design Education, v. 32, n. 2, p. 190-202, jun. 2013.

DORST, K. The core of "design thinking" and its application. Design Studies, v. 32, p. 521-232, 2011.

EXAME. QS World University Rankings 2019: Universidade de São Paulo atinge novo recorde em ranking internacional. Disponível em: https://exame.abril.com.br/negocios/releases/qs-worlduniversity-rankings-2019-universidade-de-sao-paulo-atinge-novo-recorde-em-ranking-

internacional. Acesso em: 12 jun. 2018.

HEIJDE, R. V. How Design Thinking Turned One Hospital into a Bright and Comforting. Harvard Business School Publishing Corporation, p. 2-6, 2016.

DRESCH, A.; LACERDA, D. P.; ANTUNES JR, J. A. V. Design Science Research. Cham: Springer International Publishing, 2015.

JOHANSSON-SKÖLDBERG, U.; WOODILLA, J.; ÇETINKAYA, M. Design Thinking: Past, Present and Possible Futures. Creativity and Innovation Management, v. 22, n. 2, p. 121-146, 2013.

KATHERINE, J. Meaningful Innovation: Ethnographic Potential in the Startup and Venture Capital Spheres. Ethnographic Praxis in Industry Conference Proceedings, p. 175-200, 2016.

KIMBELL, L. Rethinking Design Thinking: Part I. Design and Culture, v. 3, n. 3, p. 285-306, 2011.

LEAVY, B. Design thinking - a new mental model of value innovation. Strategy \& Leadership, v. 38, n. 3, p. 5-14, 2010.

LIEDTKA, J. Innovative ways companies are using design thinking. Strategy \& Leadership, v. 42, n. 2, p. 40-45, 2014.

LIEDTKA, J. Learning to use design thinking tools for successful innovation. Strategy \& Leadership, v. 39, n. 5, p. 13-19, 6 set. 2011. 
LIEDTKA, J.; OGILVIE, T. Designing for Growth: A Design Thinking Tool Kit for Managers. New York: Columbia University Press, 2011.

LUPTON, E. Graphic Design Thinking: Beyond Brainstorming. New York: Princeton Architectural Press, 2011.

MELLES, G.; HOWARD, Z.; THOMPSON-WHITESIDEC, S. Teaching design thinking: Expanding horizons in design education. Procedia - Social and Behavioral Sciences, v. 31, p. 162-166, 2012.

MUELLER, R. M.; THORING, K. Design Thinking Vs Lean Startup: A Comparison of Two Userdriven Innovation Strategies. Proceedings of 2012 International Design Management Research Conference.

Anais...2012.

ORTHEL, B. D. Implications of Design Thinking for Teaching, Learning, and Inquiry. Journal of Interior Design, v. 40, n. 3, p. 1-20, 2015.

OWEN, C. L. Design research: building the knowledge base. Design Studies, v. 19, n. 1, p. 9-20, 1998. PRODANOV, C. C.; FREITAS, E. C. Metodologia do Trabalho Científico: Métodos e Técnicas da Pesquisa e do Trabalho Acadêmico. 2. ed. - Novo Hamburgo: Feevale, 2013.

QS. QS World University Rankings. Disponível em: http://www.qs.com/rankings. Acesso em: 15 jul. 2017.

SHAPIRA, H.; KETCHIE, A.; NEHE, M. The integration of Design Thinking and Strategic Sustainable Development. Journal of Cleaner Production, v. 140, p. 277-287, 2017.

SIMON, H. A. The sciences of the artificial. Cambridge: MIT Press, 1996.

TSCHIMMEL, K. Design Thinking as an effective Toolkit for Innovation. XXIII ISPIM Conference: Action for Innovation: Innovating from Experience, p. 1-20, 2012.

TSCHIMMEL, K. Designer ou Design Thinker: reflexão sobre Conceitos. Arte e Design, v. 5, p. 159165, 2014.

UCL. MSIN201P: Strategy by design. Disponível em: https://www.mgmt.ucl.ac.uk/module/msin201p-strategy-design. Acesso em: 15 jul. 2017.

WOUDHUYSEN, J. The craze for design thinking: Roots, a critique, and toward an alternative. Design Principles and Practices, v. 5, p. 235-248, 2011. 